Annales Academiæ Scientiarum Fennicæ

Mathematica

Volumen 36, 2011, 245-260

\title{
ISODIAMETRIC INEQUALITY IN CARNOT GROUPS
}

\author{
Séverine Rigot \\ Université de Nice Sophia Antipolis, UMR CNRS 6621, Laboratoire J. A. Dieudonné \\ Parc Valrose, 06108 Nice Cedex 02, France; rigot@unice.fr
}

\begin{abstract}
The classical isodiametric inequality in the Euclidean space says that balls maximize the volume among all sets with a given diameter. We consider in this paper the case of Carnot groups. We prove that for any non abelian Carnot group equipped with a Haar measure one can find a homogeneous distance for which this fails to hold. We also consider Carnot-Carathéodory distances and prove that this also fails for these distances as soon as there are length minimizing curves that stop to be minimizing in finite time. Next we study some connections with the comparison between Hausdorff and spherical Hausdorff measures, rectifiability and the generalized $1 / 2$-Besicovitch conjecture, giving in particular some cases where this conjecture fails.
\end{abstract}

\section{Introduction}

The classical isodiametric inequality in the Euclidean space $\mathbf{R}^{n}$ says that balls maximize the volume among all sets with prescribed diameter,

$$
\mathscr{L}^{n}(A) \leq 2^{-n} \alpha_{n}(\operatorname{diam} A)^{n}, \quad A \subset \mathbf{R}^{n},
$$

where $\mathscr{L}^{n}$ denotes the $n$-dimensional Lebesgue measure and $\alpha_{n}$ the Lebesgue measure of the unit ball in $\mathbf{R}^{n}$. This gives non trivial information about the geometry of the Euclidean space and about the relation between the Euclidean metric and the Lebesgue measure. In particular a well-known consequence of the isodiametric inequality is the following relation between the $n$-dimensional Lebesgue measure and the $n$-dimensional Haudorff measure defined with respect to the Euclidean distance,

$$
\mathscr{L}^{n}=2^{-n} \alpha_{n} \mathscr{H}^{n}
$$

where $\mathscr{H}^{n}(A)=\lim _{\delta \downarrow} \inf \left\{\sum_{i}\left(\operatorname{diam} A_{i}\right)^{n} ; A \subset \bigcup_{i} A_{i}, \operatorname{diam} A_{i} \leq \delta\right\}$.

More generally when working on analysis on metric spaces it is a natural question to ask in what kind of settings one can have some - and what - type of isodiametric inequalities and what kind of properties can be deduced from this information.

In the present paper we are interested in Carnot groups equipped with homogeneous distances. A Carnot group is a connected and simply connected nilpotent Lie group whose Lie algebra admits a stratification. We denote by $Q$ its homogeneous dimension. It can be equipped with a natural family of dilations. A distance $d$ on a Carnot group $G$ is called homogeneous if it induces the topology of the group, is left invariant and one-homogeneous with respect to the dilations. See Section 2 for the definitions. The simplest examples of non abelian Carnot groups are the Heisenberg groups. From a metric point of view, natural measures on $(G, d)$ are given by the $Q$-dimensional Hausdorff and spherical Hausdorff measures. They turn moreover out to be non trivial left invariant measures, hence Haar measures of the group, and

doi:10.5186/aasfm.2011.3615

2010 Mathematics Subject Classification: Primary 28A75; Secondary 53C17.

Key words: Isodiametric inequality, homogeneous groups, densities. 
$Q$-homogeneous with respect to the dilations, so that they are also well-behaved and natural measures to be considered with respect to the structures the group is endowed with.

We will use throughout this paper the following general definitions and conventions about Hausdorff and spherical Hausdorff measures. Given a metric space $(M, d)$ and a non negative number $n$, we denote by $\mathscr{H}_{d}^{n}$ the $n$-dimensional Hausdorff measure given by

$$
\mathscr{H}_{d}^{n}(A)=\lim _{\delta \downarrow 0} \inf \left\{\sum_{i}\left(\operatorname{diam} A_{i}\right)^{n} ; A \subset \bigcup_{i} A_{i}, \operatorname{diam} A_{i} \leq \delta\right\},
$$

and by $\mathscr{S}_{d}^{n}$ the $n$-dimensional spherical Hausdorff measure,

$$
\mathscr{S}_{d}^{n}(A)=\lim _{\delta \downarrow 0} \inf \left\{\sum_{i}\left(\operatorname{diam} B_{i}\right)^{n} ; A \subset \bigcup_{i} B_{i}, B_{i} \text { ball, } \operatorname{diam} B_{i} \leq \delta\right\} .
$$

For a Carnot group equipped with a homogeneous distance $(G, d)$ we will more specifically take as a choice of a reference measure the $Q$-dimensional spherical Hausdorff measure $\mathscr{S}_{d}^{Q}$. A first fact supporting our choice is that

$$
\mathscr{S}_{d}^{Q}(B)=(\operatorname{diam} B)^{Q} \text { for any metric ball } B \text { in }(G, d),
$$

see Proposition 2.1. Moreover, it turns out that the isodiametric problem can be rephrased in simple terms as we shall explain now.

Going back to the isodiametric problem one seeks for the maximal possible value of the measure of sets with a given diameter. Using dilations this can be rephrased in our context into the problem of finding the maximal possible value of the ratio $\mathscr{S}_{d}^{Q}(A) /(\operatorname{diam} A)^{Q}$ among all subsets $A \subset G$ with positive and finite diameter. We denote by $C_{d}$ this value and call it the isodiametric constant,

$$
C_{d}=\sup \left\{\frac{\mathscr{S}_{d}^{Q}(A)}{(\operatorname{diam} A)^{Q}} ; 0<\operatorname{diam} A<+\infty\right\} .
$$

It obviously follows that one has the following inequality,

$$
\mathscr{S}_{d}^{Q}(A) \leq C_{d}(\operatorname{diam} A)^{Q}, \quad A \subset G,
$$

where $0<C_{d}<+\infty$ is by definition the best possible constant in the right-hand side. Since $\mathscr{S}_{d}^{Q}(B)=(\operatorname{diam} B)^{Q}$ for balls $B$, one has actually $C_{d} \geq 1$. Hence the best possible inequality one can expect is the following,

$$
\mathscr{S}_{d}^{Q}(A) \leq(\operatorname{diam} A)^{Q}, \quad A \subset G,
$$

that we call the sharp isodiametric inequality.

We are interested in this paper in the question to know whether the sharp isodiametric inequality holds, or equivalently if balls realize the supremum in the right-hand side of (1.1), in Carnot groups equipped with some specific homogeneous distances. Note that it is not difficult to see that in our context one can always find sets realizing the supremum in (1.1). We shall prove this fact for sake completeness, see Theorem 3.1. The isodiametric problem can thus be pursued with the question of finding explicitly which are these sets and this will be explored in forthcoming works (see [14]).

In the present paper we first prove that given a non abelian Carnot group $G$ one can always find some homogeneous distance on $G$, namely $d_{\infty}$-distances (see 2.2.1), for which the sharp isodiametric inequality does not hold, see Theorem 3.4. For the 
specific class of $H$-type groups equipped with the gauge distance (see 2.2.3) we prove with a similar argument that (SII) does not hold either, see Theorem 3.5. Going back to general Carnot groups, another kind of natural homogeneous distances are the socalled Carnot-Carathéodory (or sub-Riemannian) distances (see 2.2.2). Equipped with such a distance a Carnot group becomes a geodesic space. We prove that the sharp isodiametric inequality also fails in that case provided there are length minimizing curves that stop to be minimizing in finite time, see Theorem 3.6. This property holds true in particular in the Heisenberg groups and more generally in $H$-type groups.

We will then investigate some consequences of these results. One of our initial motivations in the study of the isodiametric problem was indeed some connections with geometric measure theoretic properties of the space and in particular rectifiability.

First we explicitly state and prove that in our context the comparison between the $Q$-dimensional Hausdorff and spherical Hausdorff measures can be related to the isodiametric problem in the following way,

$$
\mathscr{S}_{d}^{Q}=C_{d} \mathscr{H}_{d}^{Q}
$$

see Proposition 2.3. It follows that

$$
\text { (SII) holds in }(G, d) \Longleftrightarrow \mathscr{H}_{d}^{Q}=\mathscr{S}_{d}^{Q} \text {. }
$$

As a consequence we immediately get that in the cases we are more specifically interested in and where (SII) does not hold, the measures $\mathscr{H}_{d}^{Q}$ and $\mathscr{S}_{d}^{Q}$ do not coincide.

As another consequence of the non-validity of the sharp isodiametric inequality for at least one homogeneous distance, we recover the fact that any non abelian Carnot group equipped with a homogeneous distance and with homogeneous dimension $Q$ is purely $Q$-unrectifiable, see Subsection 4.1. This was already known, see e.g. [1], [15]. Existing proofs of this fact, and more generally of related facts, basically involve some algebraic properties of some subgroups of a Carnot group. The point here is that we give a different proof that uses only purely metric arguments.

Finally we investigate some connections with the Besicovitch 1/2-problem. First we will note that the density constant $\sigma_{Q}(G, d)$ (see Subsection 4.2 for the definition) of a non abelian Carnot group $G$ with homogeneous dimension $Q$ and equipped with a homogeneous distance $d$ satisfies $\sigma_{Q}(G, d)=C_{d}^{-1}$, see Theorem 4.2. In particular, $\sigma_{Q}(G, d)<1$ whenever the sharp isodiametric inequality does not hold in $(G, d)$. The validity of the bound $\sigma_{n}(M, d) \leq 1 / 2$ for any separable metric space $(M, d)$, which was conjectured long ago by Besicovitch for the one-dimensional density constant in $\mathbf{R}^{2}$ (see [4]), is known as the generalized Besicovitch 1/2-problem. We give here some counterexamples to this conjecture in the case of the Heisenberg groups equipped with their natural $d_{\infty}$-distance as well as with the Carnot-Carathéodory distance, see Theorem 4.3 and Theorem 4.4 .

The paper is organized as follows. In Section 2 we recall the definition of a Carnot group and we introduce the homogeneous distances on these groups we are interested in. Next in this section we state and prove some general facts about $Q$-dimensional Hausdorff and spherical Hausdorff measures on these groups equipped with homogeneous distances as mentionned before. Section 3 is devoted to the isodiametric 
problem itself. We give in this section the proof that the sharp isodiametric inequality does not hold in the cases mentionned above. Finally we investigate in Section 4 the consequences of this fact described at the end of this introduction.

\section{Carnot groups}

2.1. Carnot groups. A Carnot group $G$ is a connected and simply connected nilpotent Lie group whose Lie algebra $\mathscr{G}$ admits a stratification,

$$
\mathscr{G}=\bigoplus_{j=1}^{k} V_{j},\left[V_{1}, V_{j}\right]=V_{j+1}, V_{k} \neq\{0\}, V_{k+1}=\{0\},
$$

for some integer $k \geq 1$ called the step of the stratification.

The exponential map exp: $\mathscr{G} \rightarrow G$ is then a global diffeomorphism and the group law is given by the Campbell-Hausdorff formula,

$$
\exp X \cdot \exp Y=\exp H(X, Y),
$$

where $H(X, Y)=X+Y+[X, Y] / 2+\cdots$ where the dots indicate terms of order $\geq 3$ (the exact formula for $H$ may be found in [5, II.6.4]). We will denote by 0 the unit element of the group.

We denote by

$$
Q=\sum_{j=1}^{k} j \operatorname{dim} V_{j}
$$

the homogeneous dimension of $G$.

A natural family of dilations on $\mathscr{G}$ is given by $\delta_{\lambda}\left(\sum_{j=1}^{k} Y_{j}\right)=\sum_{j=1}^{k} \lambda^{j} Y_{j}, Y_{j} \in V_{j}$, $\lambda>0$. The maps $\exp \circ \delta_{\lambda} \circ \exp ^{-1}$ are group automorphisms of $G$. We shall denote them also by $\delta_{\lambda}$ and call them dilations on $G$.

We refer to [8] for a more detailed presentation of Carnot, and more generally homogeneous, groups.

2.2. Homogeneous distances. A distance $d$ on a Carnot group $G$ is called homogeneous if it induces the topology of the group, is left invariant,

$$
d(x \cdot y, x \cdot z)=d(y, z)
$$

for all $x, y, z \in G$, and one-homogeneous with respect to the dilations,

$$
d\left(\delta_{\lambda}(y), \delta_{\lambda}(z)\right)=\lambda d(y, z)
$$

for all $y, z \in G$ and $\lambda>0$.

A Carnot group equipped with a homogeneous distance is a separable and complete metric space in which closed bounded sets are compact. We also explicitly note that in this context the diameter of a ball is given by twice its radius. There are many ways to define homogeneous distances on a Carnot group. We shall see some examples below. As a general fact we also mention that any two homogeneous distances on a Carnot group are bilipschitz equivalent.

2.2.1. $d_{\infty}$-distances. The first kind of homogeneous distances we will consider in this paper is the class of so-called $d_{\infty}$-distances. They can be defined in the following way. First one chooses a basis $\left(X_{1}, \ldots, X_{n}\right)$ of $\mathscr{G}$ adapted to the stratification, i.e., $\left(X_{h_{j-1}+1}, \ldots, X_{h_{j}}\right)$ is a basis of $V_{j}$ where $h_{0}=0$ and $h_{j}=\operatorname{dim} V_{1}+\cdots+\operatorname{dim} V_{j}$. Next one defines an Euclidean norm $\|\cdot\|$ on $\mathscr{G}$ by declaring the $X_{j}$ 's orthonormal. 
Then one fix some positive coefficients $c_{j}$ so that $\|H(Y, Z)\|_{\infty} \leq\|Y\|_{\infty}+\|Z\|_{\infty}$ where $\|Y\|_{\infty}=\max _{j} c_{j}\left\|Y_{j}\right\|^{1 / j}$ whenever $Y=Y_{1}+\cdots+Y_{k}, Y_{j} \in V_{j}$. It turns out that one can always find such a family of coefficients (see e.g. [9]). We then set

$$
\|x\|_{\infty}=\left\|\exp ^{-1} x\right\|_{\infty} \quad \text { and } \quad d_{\infty}(x, y)=\left\|x^{-1} \cdot y\right\|_{\infty} .
$$

It is easy to check that $d_{\infty}$ is a homogeneous distance on $G$.

2.2.2. Carnot-Carathéodory distances. The second kind of homogeneous distances we wish to consider are the so-called Carnot-Carathéodory, also known as sub-Riemannian, distances which we denote by $d_{c}$. We fix a left invariant Riemannian metric $g$ on $G$ and we set

$$
d_{c}(x, y)=\inf \left\{\operatorname{length}_{g}(\gamma) ; \gamma \text { horizontal curve joining } x \text { to } y\right\}
$$

where a curve is said to be horizontal if it is absolutely continuous and such that at a.e. every point, its tangent vector belongs to the so-called horizontal subbundle of the tangent bundle whose fiber at some point $x$ is given by $\operatorname{span}\left\{X(x) ; X \in V_{1}\right\}$ when identifying elements in $V_{1}$ with left invariant vector fields. More generally one actually only needs a scalar product on the first layer $V_{1}$ of the stratification to define the related Carnot-Carathéodory distance in a similar way.

Recall that by Chow's theorem any two points can be joined by a horizontal curve and $d_{c}$ turns indeed out to be distance. One can also easily check that it is homogeneous.

An important feature of Carnot-Carathéodory distances that makes them natural distances from the geometric point of view is that, endowed with such a distance, a Carnot group becomes a geodesic space, i.e., for all $x, y \in G$, there exists a-socalled length minimizing - curve $\gamma \in C([a, b], G)$ such that $\gamma(a)=x, \gamma(b)=y$ and $d_{c}(x, y)=l_{d_{c}}(\gamma)$ where

$$
l_{d_{c}}(\gamma)=\sup _{N \in \mathbf{N}^{*}} \sup _{a=t_{0} \leq \cdots \leq t_{N}=b} \sum_{i=0}^{N-1} d_{c}\left(\gamma\left(t_{i}\right), \gamma\left(t_{i+1}\right)\right) .
$$

Up to a suitable reparameterization, one can - and we will henceforth always assume that - rectifiable curves $\gamma \in C([a, b], G)$, i.e., curves with $l_{d_{c}}(\gamma)<+\infty$, have constant speed, i.e., $(b-a) l_{d_{c}}\left(\gamma_{\mid\left[s, s^{\prime}\right]}\right)=\left(s^{\prime}-s\right) l_{d_{c}}(\gamma)$ for all $s<s^{\prime} \in[a, b]$.

For $x \in G$, we denote by $\mathscr{C}(x)$ the set of points $y \in G, y \neq x$, such that one can find a length minimizing curve $\gamma:[a, b] \rightarrow G$ from $x$ to $y$ which is no more length minimizing after reaching $y$. In other words, if $T>0$ and $c:[a, b+T] \rightarrow G$ is a rectifiable curve such that $c_{\mid[a, b]}=\gamma$ then $c$ is not length minimizing on $[a, b+T]$. We note that due to the left invariance of the distance $d_{c}$, if $\mathscr{C}(x) \neq \emptyset$ for some $x \in G$ then $\mathscr{C}(x) \neq \emptyset$ for all $x \in G$.

We say that $\left(G, d_{c}\right)$ satifies assumption $(\mathscr{C})$ if

$$
\mathscr{C}(x) \neq \emptyset \text { for some, and hence all, } x \in G .
$$

Note also that due to the homogeneity of $d_{c}$, if $\left(G, d_{c}\right)$ satifies assumption $(\mathscr{C})$, then for all $x \in G$ and $\rho>0$, one can find $y \in \mathscr{C}(x)$ such that $d_{c}(x, y)=\rho$.

Heisenberg groups (see e.g. Section 4 for the description of one model for these groups) and more generally $H$-type groups (see below for the definition) satisfy assumption $(\mathscr{C})$ (see e.g. [10], [2], resp. [13], [17], for an explicit description of length minimizing curves in Heisenberg, resp. $H$-type, groups). For general Carnot groups 
it is to our knowledge a delicate question to know whether assumption $(\mathscr{C})$ is satisfied or not. This question is in particular related to delicate issues about length minimizing curves in sub-Riemannian geometry.

We will prove in this paper that the sharp isodiametric inequality does not hold in $\left(G, d_{c}\right)$ as soon as $\left(G, d_{c}\right)$ satifies assumption $(\mathscr{C})$.

2.2.3. $H$-type groups and gauge distance. We recall here the definition of $H$-type groups. These Carnot groups are classical groups concerning many aspects of analysis, in particular Harmonic Analysis and PDE's, on homogeneous groups. We will prove the non validity of the sharp isodiametric inequality for that class of groups equipped with the gauge distance.

Let $\mathscr{G}$ be a Lie algebra equipped with a scalar product and which can be decomposed in a non trivial orthogonal direct sum, $\mathscr{G}=\mathscr{V} \oplus \mathscr{Z}$, where $[\mathscr{V}, \mathscr{V}] \subset \mathscr{Z}$ and $[\mathscr{V}, \mathscr{Z}]=[\mathscr{Z}, \mathscr{Z}]=\{0\}$. We define the linear map $J: \mathscr{Z} \rightarrow$ End $\mathscr{V}$ by $\left\langle J(Z) X, X^{\prime}\right\rangle=\left\langle Z,\left[X, X^{\prime}\right]\right\rangle$ for all $X, X^{\prime} \in \mathscr{V}$ and $Z \in \mathscr{Z}$. We say that $\mathscr{G}$ is a $H$-type algebra if for all $Z \in \mathscr{Z}$,

$$
J(Z)^{2}=-\|Z\|^{2} \mathrm{Id} .
$$

Note that in such a case $[\mathscr{V}, \mathscr{V}]=\mathscr{Z}$ so that $\mathscr{G}$ is a stratified Lie algebra of step two. For more details about $H$-type algebras we refer to e.g. [11]. A Carnot group is said to be a $H$-type group if its Lie algebra is of $H$-type.

This class of groups gives a natural generalization of the Heisenberg groups which correspond to the case where $\operatorname{dim} \mathscr{Z}=1$.

The gauge distance $d_{g}$ on a $H$-type group $G$ is defined in the following way. One defines a homogeneous norm on $G$ by

$$
\|\exp (X+Z)\|_{g}=\left(\|X\|^{4}+16\|Z\|^{2}\right)^{1 / 4}
$$

for all $X \in \mathscr{V}$ and $Z \in \mathscr{Z}$ where the norm in the right-hand side is the norm induced by the given scaler product on $\mathscr{G}$. By [6], this norm satisfies $\|x \cdot y\|_{g} \leq\|x\|_{g}+\|y\|_{g}$ for all $x, y \in G$, and one then defines the gauge distance between any two points $x$, $y \in G$ by

$$
d_{g}(x, y)=\left\|x^{-1} \cdot y\right\|_{g} .
$$

which is clearly homegeneous.

2.3. Hausdorff measures. As a classical fact the Hausdorff dimension of a Carnot group equipped with a homogeneous distance $(G, d)$ coincides with its homogeneous dimension $Q$. Moreover both measures $\mathscr{H}_{d}^{Q}$ and $\mathscr{S}_{d}^{Q}$ (see Section 1 for the definitions and conventions about Hausdorff measures) are left invariant measures which give positive and finite measure to any ball with positive and finite diameter and hence are Haar measures of the group. These measures are also $Q$-homogeneous with respect to the dilations of the group,

$$
\mathscr{H}_{d}^{Q}\left(\delta_{\lambda}(A)\right)=\lambda^{Q} \mathscr{H}_{d}^{Q}(A), \quad \mathscr{S}_{d}^{Q}\left(\delta_{\lambda}(A)\right)=\lambda^{Q} \mathscr{H}_{d}^{Q}(A),
$$

for all $A \subset G$ and $\lambda>0$. As a consequence any Haar measure $\mu$ of the group is $Q$-homogeneous as well and in particular we have $\mu(B)=C(\operatorname{diam} B)^{Q}$ for any ball $B$ in $(G, d)$ and for some constant $C>0$ which depends only on the homogeneous distance $d$ and the Haar measure $\mu$. As already mentionned in the introduction we will take as a reference measure in $(G, d)$ the measure $\mathscr{S}_{d}^{Q}$. The first observation 
supporting this choice is the following fact which gives in our context the exact - and particularly simple - value of the $\mathscr{S}_{d}^{Q}$-measure of a ball.

Proposition 2.1. Let $(G, d)$ be a Carnot group equipped with a homogeneous distance. Let $Q$ denote its homogeneous dimension. Then

$$
\mathscr{S}_{d}^{Q}(B)=(\operatorname{diam} B)^{Q}
$$

for any ball $B$ in $(G, d)$.

This follows from classical covering arguments and might be well-known as well although to our knowledge not explicitly stated that way in the literature. We give a proof below for sake of completeness. We first recall the general covering arguments to be used. Let $\mathscr{F}$ be a family of subsets of $G$. We say that $\mathscr{F}$ covers a set $A$ finely if for all $a \in A$ and all $\varepsilon>0$, there exists $F \in \mathscr{F}$ such that $a \in F$ and $\operatorname{diam} F \leq \varepsilon$. We say that $\mathscr{F}$ is adequate for a set $A$ if for any open set $U$ there exists a countable disjointed subfamily $\tilde{\mathscr{F}}$ of $\mathscr{F}$ such that $\bigcup_{F \in \tilde{\mathscr{F}}} F \subset U$ and $\mu\left((U \cap A) \backslash \bigcup_{F \in \tilde{\mathscr{F}}} F\right)=0$ where $\mu$ denotes any Haar measure of the group. If $F \in \mathscr{F}$, we set $\hat{F}=\bigcup\{T \in \mathscr{F} ; T \cap F \neq \emptyset, \operatorname{diam}(T) \leq 2 \operatorname{diam}(F)\}$.

Theorem 2.2. [7, Chapter 2.8] Assume that all sets in $\mathscr{F}$ are closed and bounded and that $\mu(F)>0$ for all $F \in \mathscr{F}$. If $\mathscr{F}$ covers a set $A$ finely and if there exists $\tau \geq 1$ such that $\mu(\hat{F}) \leq \tau \mu(F)$ for all $F \in \mathscr{F}$, then $\mathscr{F}$ is adequate for $A$.

Proof of Proposition 2.1. Let $\nu$ denote the Haar measure of the group normalized in such a way that $\nu(B)=(\operatorname{diam} B)^{Q}$ for any ball $B$ in $(G, d)$. It will actually follow from the proposition that $\mathscr{S}_{d}^{Q}=\nu$. Let $B$ be a ball in $(G, d)$. Let $\varepsilon>0$ be fixed and $\left(B_{i}\right)_{i}$ be a countable family of balls such that $B \subset \bigcup_{i} B_{i}$ and $\sum_{i}\left(\operatorname{diam} B_{i}\right)^{Q} \leq$ $\mathscr{S}_{d}^{Q}(B)+\varepsilon$. We have

$$
\nu(B) \leq \sum_{i} \nu\left(B_{i}\right)=\sum_{i}\left(\operatorname{diam} B_{i}\right)^{Q} \leq \mathscr{S}_{d}^{Q}(B)+\varepsilon .
$$

Since this is true for all $\varepsilon>0$, we get that $(\operatorname{diam} B)^{Q}=\nu(B) \leq \mathscr{S}_{d}^{Q}(B)$.

Conversely, let $\delta>0$ be fixed and $\mathscr{F}$ denote the family of closed balls in $(G, d)$ with positive diameter less than $\delta$. Let $B$ be an open ball. Then $\mathscr{F}$ covers $B$ finely and satisfies the assumptions of Theorem 2.2. Hence $\mathscr{F}$ is adequate for $B$ and one can find a countable disjointed subfamily $\tilde{\mathscr{F}} \subset \mathscr{F}$ such that $\bigcup_{F \in \tilde{F}} F \subset B$ and $\mathscr{S}_{d}^{Q}\left(B \backslash \bigcup_{F \in \tilde{\mathscr{F}}} F\right)=0$. It follows that

$$
\mathscr{S}_{d, \delta}^{Q}\left(\bigcup_{F \in \tilde{\mathscr{F}}} F\right) \leq \sum_{F \in \tilde{\mathscr{F}}}(\operatorname{diam} F)^{Q}=\nu\left(\bigcup_{F \in \tilde{\mathscr{F}}} F\right) \leq \nu(B)
$$

where $\mathscr{S}_{d, \delta}^{Q}(A)=\inf \left\{\sum_{i}\left(\operatorname{diam} B_{i}\right)^{Q} ; A \subset \bigcup_{i} B_{i}, B_{i}\right.$ ball, diam $\left.B_{i} \leq \delta\right\}$ for any $A \subset$ $G$. We have $\mathscr{S}_{d, \delta}^{Q}(B) \leq \mathscr{S}_{d, \delta}^{Q}\left(\bigcup_{F \in \tilde{\mathscr{F}}} F\right)+\mathscr{S}_{d, \delta}^{Q}\left(B \backslash \bigcup_{F \in \tilde{\mathscr{F}}} F\right)$ and $\mathscr{S}_{d, \delta}^{Q}\left(B \backslash \bigcup_{F \in \tilde{\mathscr{F}}} F\right) \leq$ $\mathscr{S}_{d}^{Q}\left(B \backslash \bigcup_{F \in \tilde{F}} F\right)=0$. It follows that

$$
\mathscr{S}_{d, \delta}^{Q}(B) \leq \nu(B) .
$$

Letting $\delta \downarrow 0$, we get that $\mathscr{S}_{d}^{Q}(B) \leq \nu(B)$.

Hence the claim follows for any open, and then also automatically for any closed, ball. 
Next we note and explicitly prove that the ratio between $\mathscr{H}_{d}^{Q}$ and $\mathscr{S}_{d}^{Q}$ can be related to the isodiametric constant $C_{d}$ (see (1.1) for the definition of $C_{d}$ ).

Proposition 2.3. Let $(G, d)$ be a Carnot group equipped with a homogeneous distance. Let $Q$ denote its homogeneous dimension. Then

$$
\mathscr{S}_{d}^{Q}=C_{d} \mathscr{H}_{d}^{Q}
$$

Proof. The proof is very similar to the proof of of Proposition 2.1. First we note that it is enough to prove that $\mathscr{S}_{d}^{Q}(U)=C_{d} \mathscr{H}_{d}^{Q}(U)$ for any open set $U$ with finite Haar measure.

Let $\varepsilon>0$ be fixed and $\left(U_{i}\right)_{i}$ be a countable family of bounded sets such that $U \subset \bigcup_{i} U_{i}$ and $\sum_{i}\left(\operatorname{diam} U_{i}\right)^{Q} \leq \mathscr{H}_{d}^{Q}(U)+\varepsilon$. We have

$$
\mathscr{S}_{d}^{Q}(U) \leq \sum_{i} \mathscr{S}_{d}^{Q}\left(U_{i}\right) \leq \sum_{i} C_{d}\left(\operatorname{diam} U_{i}\right)^{Q} \leq C_{d}\left(\mathscr{H}_{d}^{Q}(U)+\varepsilon\right) .
$$

Since this is true for all $\varepsilon>0$, we get that $\mathscr{S}_{d}^{Q}(U) \leq C_{d} \mathscr{H}_{d}^{Q}(U)$.

Conversely, for any subset $A$ of $G$ with $0<\operatorname{diam} A<+\infty$ we set $C(A)=$ $\mathscr{S}_{d}^{Q}(A) /(\operatorname{diam} A)^{Q}$. We prove that $C(A) \mathscr{H}_{d}^{Q}(U) \leq \mathscr{S}_{d}^{Q}(U)$. The required inequality $C_{d} \mathscr{H}_{d}^{Q}(U) \leq \mathscr{S}_{d}^{Q}(U)$ will then follow from the definition of $C_{d}$. First we note that $C(A) \leq C(\bar{A})$ and $C\left(A_{x, \lambda}\right)=C(A)$ where $A_{x, \lambda}=x \cdot \delta_{\lambda}(A)$ with $x \in G$ and $\lambda>$ 0 . Hence, taking closure and up to a translation, one can assume with no loss of generality that $A$ is closed and $0 \in A$. We also assume that $C(A)>0$ otherwise there is nothing to prove. Let $\delta>0$ be fixed and set $\mathscr{F}=\left\{A_{x, \lambda} ; x \in G, \lambda \leq \delta / \operatorname{diam} A\right\}$. We have $x \in A_{x, \lambda}$ and $\operatorname{diam} A_{x, \lambda}=\lambda \operatorname{diam} A$ for any $x \in G$ and $\lambda>0$ hence $\mathscr{F}$ covers $U$ finely. Next we have $\operatorname{diam} \hat{A}_{x, \lambda} \leq 5 \operatorname{diam} A_{x, \lambda}$ hence $\hat{A}_{x, \lambda}$ is contained in a ball with diameter 10 diam $A_{x, \lambda}$. On the other hand it follows from Proposition 2.1 that $C(A)=\mu(A) / \mu(B)$ where $B$ is a ball with diameter diam $A$ and $\mu$ any Haar measure. Then we get that $\mu\left(\hat{A}_{x, \lambda}\right) \leq 10^{Q} \mu\left(A_{x, \lambda}\right) / C\left(A_{x, \lambda}\right)=10^{Q} \mu\left(A_{x, \lambda}\right) / C(A)$ for any Haar measure $\mu$. The family $\mathscr{F}$ thus satisfies the assumptions of Theorem 2.2. Hence $\mathscr{F}$ is adequate for $U$ and one can find a countable disjointed subfamily $\tilde{\mathscr{F}} \subset \mathscr{F}$ such that $\bigcup_{F \in \tilde{F}} F \subset U$ and $\mathscr{H}_{d}^{Q}\left(U \backslash \bigcup_{F \in \tilde{\mathscr{F}}} F\right)=0$. Arguing as in the proof of Proposition 2.1 it follows that

$$
\begin{aligned}
C(A) \mathscr{H}_{d, \delta}^{Q}(U) & =C(A) \mathscr{H}_{d, \delta}^{Q}\left(\bigcup_{F \in \tilde{F}} F\right) \leq C(A) \sum_{F \in \tilde{\mathscr{F}}}(\operatorname{diam} F)^{Q}=\sum_{F \in \tilde{\mathscr{F}}} C(F)(\operatorname{diam} F)^{Q} \\
& =\sum_{F \in \tilde{\mathscr{F}}} \mathscr{S}_{d}^{Q}(F)=\mathscr{S}_{d}^{Q}\left(\bigcup_{F \in \tilde{\mathscr{F}}} F\right) \leq \mathscr{S}_{d}^{Q}(U)
\end{aligned}
$$

where $\mathscr{H}_{d, \delta}^{Q}(E)=\inf \left\{\sum_{i}\left(\operatorname{diam} E_{i}\right)^{Q} ; E \subset \bigcup_{i} E_{i}\right.$, diam $\left.E_{i} \leq \delta\right\}$ for any $E \subset G$. Letting $\delta \downarrow 0$, we get that $C(A) \mathscr{H}_{d}^{Q}(U) \leq \mathscr{S}_{d}^{Q}(U)$ as wanted.

\section{Isodiametric problem}

This section is devoted to the isodiametric problem in Carnot groups equipped with homogeneous distances. We refer to the introduction (see Section 1) for the statement of this problem. We shall in particular follow here the conventions and notations introduced in Section 1. When not explicitly stated it is also always implicitly assumed throughout this section that the notations $G$, resp. $d$, resp. $Q$, denotes a 
Carnot group, resp. a homogeneous distance on $G$, resp. the homogeneous dimension of $G$.

We say that a set $E$ is isodiametric in $(G, d)$ if $E$ is a compact subset of $G$ which maximizes the ratio $\mathscr{S}_{d}^{Q}(A) /(\operatorname{diam} A)^{Q}$ among all $A \subset G$ with positive and finite diameter and hence satisfying

$$
\mathscr{S}_{d}^{Q}(E)=C_{d}(\operatorname{diam} E)^{Q} .
$$

See (1.1) for the definition of the isodiametric constant $C_{d}$. Note that it is not restrictive to ask isodiametric sets to be compact as the closure of any set which realizes the supremum in the right-hand side of (1.1) is a compact set that still realizes the supremum.

Theorem 3.1. Let $(G, d)$ be a Carnot group equipped with a homogeneous distance. Let $Q$ denote its homogeneous dimension. Then isodiametric sets in $(G, d)$ do exist.

Proof. Using dilations it is enough to find a compact set $E$ with $\operatorname{diam} E=1$ and such that $\mathscr{S}_{d}^{Q}(E)=\sup \left\{\mathscr{S}_{d}^{Q}(A) ; \operatorname{diam} A=1\right\}$. Let $\left(E_{i}\right)$ be a maximizing sequence of sets with diameter 1 such that

$$
\lim _{i \rightarrow+\infty} \mathscr{S}_{d}^{Q}\left(E_{i}\right)=\sup \left\{\mathscr{S}_{d}^{Q}(A) ; \operatorname{diam} A=1\right\} .
$$

Taking closure if necessary and up to a suitable translation one can assume each $E_{i}$ to be compact and contained in the closed unit ball. Then one can extract a subsequence, still denoted by $\left(E_{i}\right)$, converging in the Hausdorff distance to some compact set $E$.

The measure $\mathscr{S}_{d}^{Q}$ being a regular measure it follows that $\mathscr{S}_{d}^{Q}$ is upper semicontinuous with respect to the convergence of compact sets in the Hausdorff distance. Indeed let $\left(A_{i}\right)$ be a sequence of compact sets converging to some compact set $A$ in the Hausdorff distance. By regularity of $\mathscr{S}_{d}^{Q}$, for any $\varepsilon>0$, one can find an open set $U \supset A$ such that $\mathscr{S}_{d}^{Q}(U) \leq \mathscr{S}_{d}^{Q}(A)+\varepsilon$. On the other hand by convergence in the Hausdorff distance we have $A_{i} \subset U$ for all $i$ large enough and hence $\mathscr{S}_{d}^{Q}\left(A_{i}\right) \leq$ $\mathscr{S}_{d}^{Q}(U)$. It follows that

$$
\limsup _{i \rightarrow+\infty} \mathscr{S}_{d}^{Q}\left(A_{i}\right) \leq \mathscr{S}_{d}^{Q}(U) \leq \mathscr{S}_{d}^{Q}(A)+\varepsilon
$$

Since this holds for any $\varepsilon>0$, we finally get

$$
\limsup _{i \rightarrow+\infty} \mathscr{S}_{d}^{Q}\left(A_{i}\right) \leq \mathscr{S}_{d}^{Q}(A)
$$

as wanted.

Going back to the maximizing sequence $\left(E_{i}\right)$, it follows that

$$
\mathscr{S}_{d}^{Q}(E) \geq \lim _{i \rightarrow+\infty} \mathscr{S}_{d}^{Q}\left(E_{i}\right)=\sup \left\{\mathscr{S}_{d}^{Q}(A) ; \operatorname{diam} A=1\right\} .
$$

On the other hand, by convergence in the Hausdorff distance, we have diam $E=1$ and hence $E$ is isodiametric.

Next we give a necessary condition for a set to be isodiametric.

Lemma 3.2. Let $(G, d)$ be a Carnot group equipped with a homogeneous distance. Let $E \subset G$ be a compact set with diam $E>0$. Assume that one can find 
$x \in \partial E$ such that $d(x, y)<\operatorname{diam} E$ for all $y \in E$. Then $E$ is not isodiametric in $(G, d)$.

Proof. Let $E$ and $x \in \partial E$ be as in the statement. Since $E$ is compact, we have $\sup _{y \in E} d(x, y)<\operatorname{diam} E$. The map $z \mapsto \sup _{y \in E} d(z, y)$ being 1-Lipschitz and hence continuous, it follows that one can find a closed ball $B$ centered at $x$ with $0<\operatorname{diam} B<\operatorname{diam} E \operatorname{such}$ that $\sup _{y \in E} d(z, y)<\operatorname{diam} E$ for all $z \in B$. We set $A=E \cup B$. We have $\operatorname{diam} A=\operatorname{diam} E$. Since $x \in \partial E$ and $E$ is closed, we have that $A \backslash E$ has non empty interior and hence positive $\mathscr{S}_{d}^{Q}$-measure. Hence $\mathscr{S}_{d}^{Q}(A)>\mathscr{S}_{d}^{Q}(E)$ which proves that $E$ is not isodiametric.

Remark 3.3. One can actually prove that if $E$ is isodiametric and $x \in \partial E$ then any $y \in E$ such that $d(x, y)=\operatorname{diam} E$ belongs to the boundary of $E$. This follows essentially from the fact that the distance function from a given point is in our context an open map (recall also that isodiametric sets are assumed to be compact). We will however not use this fact in this paper.

From now on we will consider non abelian Carnot groups, i.e., Carnot groups with a stratification of step $\geq 2$. When the stratification is of step 1 , one recovers the abelian group $\left(\mathbf{R}^{n},+\right)$. Then dilations are given by the multiplication by a scalar factor and homogeneous distances are distances induced by a norm in the classical usual sense. It is well-known that the sharp isodiametric inequality holds in $\mathbf{R}^{n}$ equipped with any distance induced by a norm. On the contrary the situation becomes definitely different as soon as the stratification is of step $\geq 2$ as we shall see below.

First we prove that for any non abelian Carnot group, there exists a homogeneous distance, namely the $d_{\infty}$-distances, for which the sharp isodiametric inequality does not hold.

Theorem 3.4. Let $\left(G, d_{\infty}\right)$ be a non abelian Carnot group equipped with a homogeneous $d_{\infty}$-distance. Then closed balls in $\left(G, d_{\infty}\right)$ are not isodiametric and the sharp isodiametric inequality (SII) does not hold in $\left(G, d_{\infty}\right)$.

Proof. Let the $d_{\infty}$-distance be defined as in 2.2.1. Using left translations and dilations we only need to prove that the closed unit ball in $\left(G, d_{\infty}\right)$ is not isodiametric. Let $B$ denote the closed unit ball in $\left(G, d_{\infty}\right)$. Let $X \in V_{k}$ be such that $c_{k}\|X\|^{1 / k}=1$ and set $x=\exp X$. Then $x \in \partial B$. Let $y \in B, y=\exp \left(Y_{1}+\cdots+Y_{k}\right), Y_{j} \in V_{j}$. We have

$$
x^{-1} \cdot y=\exp (-X) \cdot \exp \left(\sum_{j=1}^{k} Y_{j}\right)=\exp \left(\sum_{j=1}^{k-1} Y_{j}+Y_{k}-X\right) .
$$

Since

$$
c_{k}^{2 k}\left\|Y_{k}-X\right\|^{2}=c_{k}^{2 k}\left\|Y_{k}\right\|^{2}+c_{k}^{2 k}\|X\|^{2}-2 c_{k}^{2 k}\left\langle Y_{k}, X\right\rangle \leq 2+2 c_{k}^{k}\left\|Y_{k}\right\| c_{k}^{k}\|X\| \leq 4,
$$

we get $d_{\infty}(x, y) \leq \max \left(1,2^{1 / k}\right)<2=\operatorname{diam} B$ and the conclusion follows from Lemma 3.2 .

Similarly the sharp isodiametric inequality (SII) does not hold in $H$-type groups equipped with the gauge distance. See 2.2.3 for the definitions of $H$-type groups and of the gauge distance. 
Theorem 3.5. Let $\left(G, d_{g}\right)$ be a $H$-type group equipped with the gauge distance. Then closed balls in $\left(G, d_{g}\right)$ are not isodiametric and the sharp isodiametric inequality (SII) does not hold in $\left(G, d_{g}\right)$.

Proof. Arguing as in the proof of Theorem 3.4 we prove that the closed unit ball in $\left(G, d_{g}\right)$ - denoted by $B$-is not isodiametric. Following the notations of 2.2 .3 we choose some $x=\exp \left(Z_{x}\right)$ with $Z_{x} \in \mathscr{Z}$ such that $\left\|Z_{x}\right\|=1 / 4$. Then if $y=\exp (X+$ $Z) \in B$ with $X \in \mathscr{V}$ and $Z \in \mathscr{Z}$, we have $\exp \left(Z_{x}\right)^{-1} \cdot \exp (X+Z)=\exp \left(X+Z-Z_{x}\right)$ hence

$$
\begin{aligned}
d_{g}(x, y)^{4} & =\|X\|^{4}+16\left\|Z-Z_{x}\right\|^{2} \\
& =\|X\|^{4}+16\|Z\|^{2}+16\left\|Z_{x}\right\|^{2}-32\left\langle Z_{x}, Z\right\rangle \leq 2\left(1+16\|Z\|\left\|Z_{x}\right\|\right) \leq 4
\end{aligned}
$$

since $y \in B$ and hence $\|X\|^{4}+16\|Z\|^{2} \leq 1$ and $\|Z\| \leq 1 / 4$. It follows that $d_{g}(x, y) \leq$ $\sqrt{2}<2=\operatorname{diam} B$ and we get the conclusion from Lemma 3.2.

Finally we investigate the case of the Carnot-Carathéodory distances. See 2.2.2 for the definition of Carnot-Carathéodory distances.

Theorem 3.6. Let $\left(G, d_{c}\right)$ be a non abelian Carnot group equipped with a Carnot-Carathéodory distance. Assume that $\left(G, d_{c}\right)$ satisfies assumption $(\mathscr{C})$. Then closed balls in $\left(G, d_{c}\right)$ are not isodiametric and the sharp isodiametric inequality (SII) does not hold in $\left(G, d_{c}\right)$.

Proof. By assumption $(\mathscr{C})$ one can find $x \in \mathscr{C}(0)$ with $d_{c}(0, x)=1$ and $\gamma:[0,1] \rightarrow$ $G$ a length minimizing curve from 0 to $x$ which is no more length minimizing after reaching $x$. Let $B$ denote the closed ball with center $x$ and radius one. We prove that $B$, and hence any closed ball, is not isodiametric in $\left(G, d_{c}\right)$. Let $y \in B$ and $c:\left[0,1+d_{c}(x, y)\right] \rightarrow G$ be such that $c_{\mid[0,1]}=\gamma$ and $c_{\mid\left[1,1+d_{c}(x, y)\right]}$ is a unit speed length minimizing curve from $x$ to $y$. Then $c$ is not length minimizing on $\left[0,1+d_{c}(x, y)\right]$ by choice of $\gamma$ hence $d_{c}(0, y)<l_{d_{c}}(c)=d_{c}(0, x)+d_{c}(x, y) \leq 2=\operatorname{diam} B$ and the conclusion follows from Lemma 3.2 .

\section{Some consequences and related problems}

4.1. Non-rectifiabilty. We recover here the fact that a non abelian Carnot group equipped with a homogeneous distance and with homogeneous dimension $Q$ is purely $Q$-unrectifiable. This is already well-known, see e.g. [1], [15] for more and further results about rectifiability in Carnot groups. The point is that we give here a different proof using only metric arguments. Recall that a metric space $(M, d)$ is said to be purely $n$-unrectifiable if one has $\mathscr{H}_{d}^{n}(f(A))=0$ for every Lipschitz map $f: A \subset \mathbf{R}^{n} \rightarrow(M, d)$ where $\mathbf{R}^{n}$ is equipped with the Euclidean distance.

Theorem 4.1. Let $(G, d)$ be a non abelian Carnot group equipped with a homogeneous distance. Let $Q$ denote its homogeneous dimension. Then $(G, d)$ is purely $Q$-unrectifiable.

Proof. The property of being purely $Q$-unrectifiable is invariant under a change of bilipschitz equivalent distance. Since all homogeneous distances are bilipschitz equivalent, one can assume that $G$ is equipped with a $d_{\infty}$-distance defined as in 2.2.1. Assume by contradiction that one can find a Lipschitz map $f: A \subset \mathbf{R}^{Q} \rightarrow\left(G, d_{\infty}\right)$ such that $\mathscr{H}_{d_{\infty}}^{Q}(f(A))>0$. One can also assume with no loss of generality that $A$ is 
bounded and hence $\mathscr{H}_{d_{\infty}}^{Q}(f(A))<+\infty$. Then one has

$$
\lim _{r \downarrow 0} \frac{\mathscr{H}_{d_{\infty}}^{Q}(f(A) \cap B(x, r))}{(2 r)^{Q}}=1
$$

for $\mathscr{H}_{d_{\infty}}^{Q}$-a.e. $x \in f(A)$ (see [12]). On the other hand it follows from Proposition 2.1, Proposition 2.3 and Theorem 3.4 that

$$
\frac{\mathscr{H}_{d_{\infty}}^{Q}(f(A) \cap B(x, r))}{(2 r)^{Q}} \leq \frac{\mathscr{H}_{d_{\infty}}^{Q}(B(x, r))}{(2 r)^{Q}}=\frac{\mathscr{H}_{d_{\infty}}^{Q}(B(x, r))}{\mathscr{S}_{d_{\infty}}^{Q}(B(x, r))}=C_{d_{\infty}}^{-1}<1
$$

which gives a contradiction.

4.2. Besicovitch 1/2-problem We investigate now some connection between the isodiametric problem and densities. Following [16] given a metric space $(M, d)$ we denote by $\sigma_{n}(M, d)$ the smallest number such that every subset $A \subset M$ of finite $\mathscr{H}_{d}^{n}$-measure having

$$
\underline{D}_{n}(A, x)>\sigma_{n}(M, d)
$$

at $\mathscr{H}_{d}^{n}$-a.e. $x \in A$ is $n$-rectifiable, where the lower $n$-density of $A$ at $x$ is given by

$$
\underline{D}_{n}(A, x)=\liminf _{r \downarrow 0} \frac{\mathscr{H}_{d}^{n}(A \cap B(x, r))}{(2 r)^{n}} .
$$

We call this number the density constant. Recall that $A$ is said $n$-rectifiable if $\mathscr{H}_{d}^{n}$ almost all of $A$ can be covered by countably many Lipschitzian images of subsets of the Euclidean space $\mathbf{R}^{n}$.

It follows from famous results of Besicovitch (see [3], [4]) that one always has $\sigma_{n}(M, d) \leq 1$. These results also imply that the fact that $\sigma_{n}(M, d)=1$ does not give any positive information about rectifiability. See [16] for a more detail account about known results on density constants.

We first note and explicitly prove that $\sigma_{Q}(G, d)<1$ whenever $d$ is a homogeneous distance on a Carnot group $G$ for which the sharp isodiametric inequality (SII) does not hold and $Q$ denotes the homogeneous dimension of $G$. More precisely

Theorem 4.2. Let $(G, d)$ be a non abelian Carnot group equipped with a homogeneous distance. Let $Q$ denote its homogeneous dimension. Then one has

$$
\sigma_{Q}(G, d)=C_{d}^{-1}
$$

and hence $\sigma_{Q}(G, d)<1$ if and only if the sharp isodiametric inequality (SII) does not hold in $(G, d)$.

Proof. Let $U \subset G$ be open. It follows from Proposition 2.1 and Proposition 2.3 that

$$
\underline{D}_{n}(U, x)=\liminf _{r \downarrow 0} \frac{\mathscr{H}_{d}^{Q}(B(x, r))}{(2 r)^{Q}}=\liminf _{r \downarrow 0} \frac{\mathscr{H}_{d}^{Q}(B(x, r))}{\mathscr{S}_{d}^{Q}(B(x, r))}=C_{d}^{-1}
$$

for all $x \in U$. Since $(G, d)$ is purely $Q$-unrectifiable (see e.g. Theorem 4.1) it follows that $\sigma_{Q}(G, d) \geq C_{d}^{-1}$. On the other hand, one obviously has

$$
\underline{D}_{n}(A, x) \leq \underline{D}_{n}(G, x)=C_{d}^{-1}
$$

for all $A \subset G$ and $x \in G$. Hence $\sigma_{Q}(G, d) \leq C_{d}^{-1}$ which concludes the proof.

Besicovitch conjectured in [4] that $\sigma_{1}\left(\mathbf{R}^{2}\right) \leq 1 / 2$. More generally the question to know whether $\sigma_{n}(M, d) \leq 1 / 2$ for any separable metric space $(M, d)$ is known as the 
generalized Besicovitch 1/2-problem. We investigate below the case of the Heisenberg groups equipped with a $d_{\infty}$-distance as well as with a Carnot-Carathéodory distance exhibiting cases where the conjecture fails.

We first describe the model we consider for the Heisenberg group $\mathbf{H}^{n}$. We identify it with $\mathbf{R}^{2 n} \times \mathbf{R}$ and denote points in $\mathbf{H}^{n}$ by $[z, t], z=\left(x_{1}, \ldots, x_{2 n}\right) \in \mathbf{R}^{2 n}, t \in \mathbf{R}$. The group law is

$$
[z, t] \cdot\left[z^{\prime}, t^{\prime}\right]=\left[z+z^{\prime}, t+t^{\prime}+2 \sum_{j=1}^{n}\left(x_{n+j} x_{j}^{\prime}-x_{j} x_{n+j}^{\prime}\right)\right] .
$$

The stratification is given by

$$
V_{1}=\operatorname{span}\left\{X_{j}, Y_{j} ; j=1, \ldots, n\right\}, V_{2}=\operatorname{span}\left\{\partial_{t}\right\},
$$

where $X_{j}=\partial_{x_{j}}+2 x_{n+j} \partial_{t}$ and $Y_{j}=\partial_{x_{n+j}}-2 x_{j} \partial_{t}$. The dilations are $\delta_{\lambda}([z, t])=$ $\left[\lambda z, \lambda^{2} t\right]$. The homogeneous dimension of $\mathbf{H}^{n}$ is $2 n+2$. The $(2 n+1)$-dimensional Lebesgue measure $\mathscr{L}^{2 n+1}$ on $\mathbf{H}^{n} \approx \mathbf{R}^{2 n} \times \mathbf{R}$ is a Haar measure of the group.

We consider first the $d_{\infty}$-distance defined with respect to the homogeneous norm $\|[z, t]\|_{\infty}=\max \left(\|z\|,|t|^{1 / 2}\right)$ where here $\|\cdot\|$ denotes the Euclidean norm in $\mathbf{R}^{2 n}$,

$$
d_{\infty}\left([z, t],\left[z^{\prime}, t^{\prime}\right]\right)=\left\|[z, t]^{-1} \cdot\left[z^{\prime}, t^{\prime}\right]\right\|_{\infty} .
$$

Theorem 4.3. The isodiametric constant $C_{d_{\infty}}$ in $\left(\mathbf{H}^{n}, d_{\infty}\right)$ satisfies $C_{d_{\infty}}<2$ and hence $\sigma_{2 n+2}\left(\mathbf{H}^{n}, d_{\infty}\right)>1 / 2$.

Proof. First we note that due to Proposition 2.1 and using dilations the isodiametric constant can be rewritten as

$$
C_{d_{\infty}}=\frac{\sup \mathscr{L}^{2 n+1}(A)}{\mathscr{L}^{2 n+1}(B)}
$$

where the supremum is taken over all compact subsets $A$ of $\left(\mathbf{H}^{n}, d_{\infty}\right)$ with $\operatorname{diam} A=2$ and $B$ denotes the closed unit ball in $\left(\mathbf{H}^{n}, d_{\infty}\right)$. We have $B=\left\{[z, t] \in \mathbf{H}^{n} ;\|z\| \leq\right.$ $1,|t| \leq 1\}$ hence

$$
\mathscr{L}^{2 n+1}(B)=2 \mathscr{L}^{2 n}(U)
$$

where $U$ denotes the closed unit Euclidean ball in $\mathbf{R}^{2 n}$ and $\mathscr{L}^{2 n}$ the $2 n$-dimensional Lebesgue measure on $\mathbf{R}^{2 n}$. Let $p: \mathbf{H}^{n} \rightarrow \mathbf{R}^{2 n}$ denote the projection defined by $p([z, t])=z$. Let $A$ be a compact subset of $\left(\mathbf{H}^{n}, d_{\infty}\right)$ with $\operatorname{diam} A=2$. The map $p$ is 1-Lipschitz from $\left(\mathbf{H}^{n}, d_{\infty}\right)$ to the Euclidean space $\left(\mathbf{R}^{2 n},\|\cdot\|\right)$ hence

$$
\mathscr{L}^{2 n}(p(A)) \leq \mathscr{L}^{2 n}(U)
$$

by the classical isodiametric inequality in $\left(\mathbf{R}^{2 n},\|\cdot\|\right)$. Next for any $z \in p(A)$, we have $\left|t-t^{\prime}\right| \leq d_{\infty}\left([z, t],\left[z, t^{\prime}\right]\right)^{2} \leq 4$ whenever $[z, t]$ and $\left[z, t^{\prime}\right] \in A$ hence $A \cap p^{-1}(\{z\})$ is contained in some segment with length at most 4. It follows from Fubini's Theorem that

$$
\mathscr{L}^{2 n+1}(A) \leq 4 \mathscr{L}^{2 n}(U)
$$

and hence

$$
C_{d_{\infty}} \leq 2
$$

Assume by contradiction that $C_{d_{\infty}}=2$ and thus (see Theorem 3.1) that one can find a compact subset $E$ in $\left(\mathbf{H}^{n}, d_{\infty}\right)$ with $\operatorname{diam} E=2$ and such that $\mathscr{L}^{2 n+1}(E)=$ $4 \mathscr{L}^{2 n}(U)$. By the argument above we know that for any $z \in p(E)$ we have $\mathscr{L}^{1}(E \cap$ 
$\left.p^{-1}(\{z\})\right) \leq 4$ where $\mathscr{L}^{1}$ denotes the one-dimensional Lebesgue measure. On the other hand, remembering (4.1), we have

$$
\int_{p(E)}\left(\mathscr{L}^{1}\left(E \cap p^{-1}(\{z\})\right)-4\right) d \mathscr{L}^{2 n}(z) \geq \mathscr{L}^{2 n+1}(E)-4 \mathscr{L}^{2 n}(U)=0 .
$$

It follows that $\mathscr{L}^{1}\left(E \cap p^{-1}(\{z\})\right)=4$ and consequently that $E \cap p^{-1}(\{z\})$ is a whole segment with length $4, E \cap p^{-1}(\{z\})=\left\{[z, t] ; t_{z}^{-} \leq t \leq t_{z}^{+}\right\}$for some $t_{z}^{-}$ and $t_{z}^{+}$such that $t_{z}^{+}-t_{z}^{-}=4$, for $\mathscr{L}^{2 n}$-a.e. $z \in p(E)$. Up to a translation one can assume that $0 \in E$ and that $E \cap p^{-1}(\{0\})=\{[0, t] ; 0 \leq t \leq 4\}$. Then the fact that $d_{\infty}\left([0,4],\left[z, t_{z}^{-}\right]\right) \leq 2$ implies in particular that $t_{z}^{-} \geq 0$ and on the other hand $d_{\infty}\left([0,0],\left[z, t_{z}^{+}\right]\right) \leq 2$ implies that $t_{z}^{+} \leq 4$ and we finally get that $t_{z}^{-}=0$ and $t_{z}^{+}=4$ for $\mathscr{L}^{2 n}$-a.e. $z \in p(E)$.

For $z=\left(x_{1}, \ldots, x_{2 n}\right) \in \mathbf{R}^{2 n}$ we consider now the half-space $H_{z}$ in $\mathbf{R}^{2 n}$ defined by $H_{z}=\left\{z^{\prime}=\left(x_{1}^{\prime}, \ldots, x_{2 n}^{\prime}\right) \in \mathbf{R}^{2 n} ; \sum_{j=1}^{n}\left(x_{n+j} x_{j}^{\prime}-x_{j} x_{n+j}^{\prime}\right)<0\right\}$. We have $z \in \partial H_{z}$. On the other hand, considering points in $p(E)$ where $p(E)$ has density one, we recall that

$$
\lim _{r \downarrow 0} \frac{\mathscr{L}^{2 n}(p(E) \cap U(z, r))}{\mathscr{L}^{2 n}(U(z, r))}=1
$$

for $\mathscr{L}^{2 n}$-a.e. $z \in p(E)$ where $U(z, r)$ denotes the (closed) ball in $\left(\mathbf{R}^{2 n},\|\cdot\|\right)$ with center $z$ and radius $r$. It follows that $\mathscr{L}^{2 n}\left(p(E) \cap H_{z}\right)>0$ for $\mathscr{L}^{2 n}$-a.e. $z \in p(E)$.

All together we finally get that one can find $z$ and $z^{\prime} \in p(E)$ such that $t_{z}^{-}=t_{z^{\prime}}^{-}=$ $0, t_{z}^{+}=t_{z^{\prime}}^{+}=4$ and $z^{\prime} \in H_{z}$. It follows that

$$
\operatorname{diam} E \geq d_{\infty}\left([z, 0],\left[z^{\prime}, 4\right]\right) \geq\left(4-2 \sum_{j=1}^{n}\left(x_{n+j} x_{j}^{\prime}-x_{j} x_{n+j}^{\prime}\right)\right)^{1 / 2}>2
$$

which gives a contradiction and concludes the proof.

We consider now the Carnot-Carthéodory distance $d_{c}$ on $\mathbf{H}^{n}$ defined in the following way. We consider the left invariant Riemannian metric $g$ on $\mathbf{H}^{n}$ that makes $\left(X_{1}, \ldots, X_{n}, Y_{1}, \ldots, Y_{n}, \partial_{t}\right)$ an orthonormal basis and we define $d_{c}$ as in 2.2.2. Explicit description of balls in $\left(\mathbf{H}^{n}, d_{c}\right)$ are well-known. We refer to, e.g., [10] or [2]. If we denote by $B$ the closed unit ball in $\left(\mathbf{H}^{n}, d_{c}\right)$ one has

$$
B=\left\{\left[\frac{\sin \varphi}{\varphi} \cdot \chi, \frac{2 \varphi-\sin (2 \varphi)}{2 \varphi^{2}}\|\chi\|^{2}\right] ; \chi \in \mathbf{R}^{2 n},\|\chi\| \leq 1, \varphi \in[-\pi, \pi]\right\} .
$$

It follows that

$$
\begin{aligned}
\mathscr{L}^{2 n+1}(B) & =4 n \alpha_{2 n} \int_{0}^{\pi} \frac{2 \varphi-\sin (2 \varphi)}{2 \varphi^{2}} \cdot\left(\frac{\sin \varphi}{\varphi}\right)^{2 n-1} \cdot\left|\left(\frac{\sin \varphi}{\varphi}\right)^{\prime}\right| d \varphi \\
& =4 n \alpha_{2 n} \int_{0}^{\pi} \frac{2 \varphi-\sin (2 \varphi)}{2 \varphi^{2}} \cdot\left(\frac{\sin \varphi}{\varphi}\right)^{2 n-1} \cdot \frac{\sin \varphi-\varphi \cos \varphi}{\varphi^{2}} d \varphi .
\end{aligned}
$$

Let $A$ be a compact subset of $\left(\mathbf{H}^{n}, d_{c}\right)$ with diam $A=2$. The projection $p$ defined as before by $p([z, t])=z$ is 1 -Lipschitz from $\left(\mathbf{H}^{n}, d_{c}\right)$ to $\left(\mathbf{R}^{2 n},\|\cdot\|\right)$ hence (4.1) still holds for $A$. Next we recall that

$$
d_{c}\left([z, t],\left[z, t^{\prime}\right]\right)=\left(\pi\left|t-t^{\prime}\right|\right)^{1 / 2}
$$


hence $A \cap p^{-1}(\{z\})$ is contained in some segment with length at most $4 / \pi$. It follows that

$$
\mathscr{L}^{2 n+1}(A) \leq 4 \pi^{-1} \alpha_{2 n} .
$$

All together this gives an upper bound for the isodiametric constant $C_{d_{c}}$ in $\left(\mathbf{H}^{n}, d_{c}\right)$,

$$
C_{d_{c}} \leq \frac{1}{n \pi}\left(\int_{0}^{\pi} \frac{2 \varphi-\sin (2 \varphi)}{2 \varphi^{2}} \cdot\left(\frac{\sin \varphi}{\varphi}\right)^{2 n-1} \cdot \frac{\sin \varphi-\varphi \cos \varphi}{\varphi^{2}} d \varphi\right)^{-1}
$$

One can compute numerically this upper bound. For $n=1$ one gets an upper bound $\leq 1.22$. Then it increases for $n$ equals 1 to 8 up to a value $\leq 1.98$. For $n=9$ the upper bound is larger than 2 and this argument leaves actually open the cases $n \geq 9$. Summing up, we get the following

Theorem 4.4. Let $n \in\{1, \ldots, 8\}$. The isodiametric constant $C_{d_{c}}$ in $\left(\mathbf{H}^{n}, d_{c}\right)$ satisfies $C_{d_{c}}<2$ and hence $\sigma_{2 n+2}\left(\mathbf{H}^{n}, d_{c}\right)>1 / 2$.

\section{References}

[1] Ambrosio, L., and B. Kirchheim: Rectifiable sets in metric and Banach spaces. - Math. Ann. 318:3, 2000, 527-555.

[2] Ambrosio, L., and S. Rigot: Optimal mass transportation in the Heisenberg group. - J. Funct. Anal. 208:2, 2004, 261-301.

[3] Besicovitch, A. S.: On the fundamental geometrical properties of linearly measurable plane sets of points. - Math. Ann. 98:1, 1928, 422-464.

[4] Besicovitch, A. S.: On the fundamental geometrical properties of linearly measurable plane sets of points (II). - Math. Ann. 115:1, 1938, 296-329.

[5] Bourbaki, N.: Eléments de mathématique. Fasc. XXXVII. Groupes et algèbres de Lie. Chapitre II: Algèbres de Lie libres. Chapitre III: Groupes de Lie. - Actualités Scientifiques et Industrielles 1349, Hermann, Paris, 1972.

[6] CyGan, J.: Subadditivity of homogeneous norms on certain nilpotent Lie groups. - Proc. Amer. Math. Soc. 83:1, 1981, 69-70.

[7] Federer, H.: Geometric measure theory. - Grundlehren Math. Wiss. 153, Springer-Verlag, New York, 1969.

[8] Folland, G. B., and E. M. Stein: Hardy spaces on homogeneous groups. - Math. Notes 28, Princeton Univ. Press, Princeton, N.J.; University of Tokyo Press, Tokyo, 1982.

[9] Franchi, B., R. Serapioni, and F. Serra Cassano: On the structure of finite perimeter sets in step 2 Carnot groups. - J. Geom. Anal. 13:3, 2003, 421-466.

[10] Gaveau, B.: Principe de moindre action, propagation de la chaleur et estimes sous elliptiques sur certains groupes nilpotents. - Acta Math. 139:1-2, 1977, 95-153.

[11] Kaplan, A.: Fundamental solutions for a class of hypoelliptic PDE generated by composition of quadratic forms. - Trans. Amer. Math. Soc. 258:1, 1980, 147-153.

[12] Kirchheim, B.: Rectifiable metric spaces: local structure and regularity of the Hausdorff measure. - Proc. Amer. Math. Soc. 121:1, 1994, 113-123.

[13] Korànyi, A.: Geometric properties of Heisenberg-type groups. - Adv. Math. 56:1, 1985, 28-38.

[14] Leonardi, G. P., S. Rigot, and D. Vittone: Isodiametric sets in the Heisenberg group. In preparation.

[15] Magnani, V.: Unrectifiability and rigidity in stratified groups. - Arch. Math. (Basel) 83:6, $2004,568-576$. 
[16] Preiss, D., and J. Tiser: On Besicovitch's 1/2-problem. - J. London Math. Soc. (2) 45:2, 1992, 279-287.

[17] Rigot, S.: Mass transportation in groups of type H. - Commun. Contemp. Math. 7:4, 2005, 509-537.

Received 12 April 2010 